\title{
Defective phagocytosis in insulin controlled diabetics: evidence for a reaction between glucose and opsonising proteins
}

\author{
NJ DAVIDSON, JM SOWDEN, J FLETCHER \\ From the Department of Haematology, City Hospital, Nottingham
}

SUMMARY Neutrophils from diabetic patients controlled with insulin showed impaired phagocytosis of a yeast, Candida guilliermondii. The defect was detected by measuring the initial rate of phagocytosis at optimal concentrations of phagocytes and organisms in autologous plasma. By mixing normal neutrophils in diabetic plasma and vice versa both cellular and plasma abnormalities were shown. The defect was reproduced by incubating normal plasma at a D-glucose concentration of $15 \mathrm{mmol} / \mathrm{l}$ for $3 \mathrm{~h}$ or for shorter periods at higher concentrations of glucose. The data suggest that defective phagocytosis is partly due to a reaction between glucose and the plasma proteins concerned with opsonisation. Defective phagocytosis may be important not only in coping with infections but also in other diabetic complications as plasma proteins are concerned with the removal of damaged or effete cells as well as foreign antigens.

Patients with diabetes mellitus are susceptible to a variety of bacterial and fungal diseases including urinary tract infection, pulmonary tuberculosis, candidal vulvo-vaginitis, soft tissue infection with staphylococci, and, possibly, Gram negative bacteraemia. ${ }^{\prime}$ Consequently, there has been considerable interest is the possibility of abnormal neutrophil function in diabetes, and defects have been reported in neutrophil adherence, ${ }^{2}$ chemotaxis, ${ }^{34}$ and microbicidal activity. ${ }^{5-7}$ Evidence for a defect in phagocytic function is conflicting, with some reports suggesting that it is normal ${ }^{389}$ and others suggesting that it is deranged..$^{510}$ Most of the abnormalities described can be attributed to ketoacidosis or a hyperosmolar state and there remains doubt as to whether phagocytosis is defective in uncomplicated diabetes.

We investigated the ability of neutrophils to phagocytose a yeast, Candida guilliermondii, and arranged the conditions so that the entire population of neutrophils was challenged. ${ }^{1 "}$ Measurements were made at the beginning of the incubation at times ignored in many previous studies. With these methods neutrophils from uncomplicated diabetic patients showed considerable impairment of phagocytosis. The defect was reproduced by incubating normal plasma at raised glucose concent-

Accepted for publication 1 March 1984 rations before testing phagocytosis, suggesting that there is a reaction between glucose and the proteins concerned with opsonisation.

\section{Material and methods}

\section{PATIENTS}

Eleven diabetic outpatients were studied. At the time of venesection the mean blood glucose concentration was $11.5 \mathrm{mmol} / \mathrm{l}( \pm 4.1 \mathrm{SD})$, range 5.4 $18.8 \mathrm{mmol} / \mathrm{l}$, and the mean glycosylated haemoglobin was $11.6 \%( \pm 1 \cdot 5 \mathrm{SD})$, range $8 \cdot 5-13.0 \%$. There was no evidence of ketoacidosis or infection. All patients were controlled with insulin. The controls were healthy volunteers with a mean blood glucose concentration of $4.6 \mathrm{mmol} / \mathrm{l}( \pm 0.8 \mathrm{SD})$, range 3.3 $6.1 \mathrm{mmol} / \mathrm{l}$, and mean glycosylated haemoglobin of $6 \cdot 2 \%( \pm 0 \cdot 9 \mathrm{SD})$, range $4 \cdot 9-8 \cdot 2 \%$.

\section{NEUTROPHILS}

Leucocytes were separated from heparinised ( 10 units $/ \mathrm{ml}$ ) venous blood by dextran sedimentation, washed twice in TC199 (Wellcome Reagents, Beckenham, England) and resuspended in TC199 at a concentration of $9 \times 10^{\circ}$ neutrophils $/ \mathrm{ml}$.

\section{PLASMA}

Autologous plasma was used as the source of opsonin. A separate sample of heparinised ( 5 units $/ \mathrm{ml}$ ) venous blood was centrifuged at $200 \mathrm{~g}$ to remove 
cells and $1000 \mathrm{~g}$ to remove platelets.

\section{GUILLIERMONDII}

C guilliermondii was cultured in Sabarauds' broth, washed twice in TC199, and suspended at a concentration of $40 \times 10^{6}$ candida $/ \mathrm{ml}$.

\section{PHAGOCYTOSIS}

Candida suspension ( $0.25 \mathrm{ml}$ volumes), plasma, and TC199 were mixed and equilibrated at $37^{\circ} \mathrm{C}$ before adding $0.25 \mathrm{ml}$ of the leucocyte suspension. After incubation at $37^{\circ} \mathrm{C} 0.1 \mathrm{ml}$ aliquots were removed at $0,5,10,15,20,30$, and $45 \mathrm{~min}$, mixed with cold $\left(4^{\circ} \mathrm{C}\right) 0.006 \mathrm{M}$ edetic acid to stop phagocytosis, and cytocentrifuge preparations stained with Leishman's stain. Phagocytosis was assessed microscopically by counting only neutrophils containing a yeast entirely within the cell and surrounded by the clear halo of a phagocytic vacuole. A total of 400 neutrophils were examined on each slide and the proportion having ingested one or more candida was called the percentage phagocytosis. By mixing normal cells with diabetic plasma and diabetic cells with normal plasma it was possible to determine whether impaired phagocytosis was due to cellular, plasma, or combined defects.

\section{GLUCOSE INCUBATIONS}

Normal plasma was preincubated at $37^{\circ} \mathrm{C}$ at varying concentrations of $D$-glucose $(15-52 \mathrm{mmol} / \mathrm{l})$ for varying times (0-180 min) before measuring phagocytosis. The control studies consisted of adding glucose immediately before measuring phagocytosis, adding the inert sugar D-mannitol in place of D-glucose, and no added sugar.

\section{STATISTICS}

The data were tested for significance by the unpaired Student's $t$ test.

\section{Results}

Comparison of neutrophils from diabetic outpatients and controls showed significant impairment of phagocytosis by the diabetics' cells at all incubation times, the largest and most significant differences were at the earliest times (Fig. 1). All the patients showed similar impairment of phagocytosis, and there was no significant correlation with blood glucose or glycosylated haemoglobin values. Defective phagocytosis was not due to the presence of more than one population of cells as there were more diabetic cells containing no candida and fewer containing 2, 3, or 4 compared with normal (Table).

In five separate experiments diabetic cells were mixed with normal plasma and normal cells with

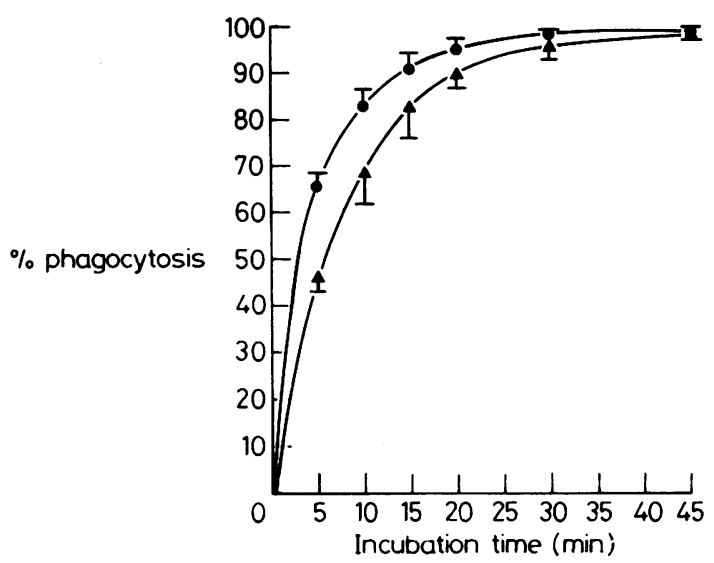

Fig. 1 Percentage phagocytosis plotted against duration of incubation for diabetics ( $\Delta$ ) and control (O) subjects. Each point represents the mean \pm 1 standard deviation.

diabetic plasma and in each case there was a significant abnormality of phagocytosis. The size of the defect due to diabetic plasma and the defect due

Distribution of candida in neutrophils of seven normal and 11 diabetic subjects after incubation for 10 min

\begin{tabular}{lll}
\hline \multirow{2}{*}{$\begin{array}{l}\text { No of candida } \\
\text { per neutrophil }\end{array}$} & \multicolumn{2}{l}{ Percentage of neutrophil population (mean $\pm S D)$} \\
\cline { 2 - 3 } & Diabetic & Normal \\
\hline 0 & $36.0 \pm 10 \cdot 1$ & $23.4 \pm 4 \cdot 0$ \\
1 & $29.4 \pm 3 \cdot 9$ & $26.6 \pm 6.4$ \\
2 & $18.7 \pm 3 \cdot 9$ & $23.0 \pm 3 \cdot 5$ \\
3 & $7 \cdot 7 \pm 3 \cdot 2$ & $13 \cdot 1 \pm 2 \cdot 0$ \\
4 & $4.6 \pm 2.9$ & $6.9 \pm 2.4$ \\
\hline
\end{tabular}

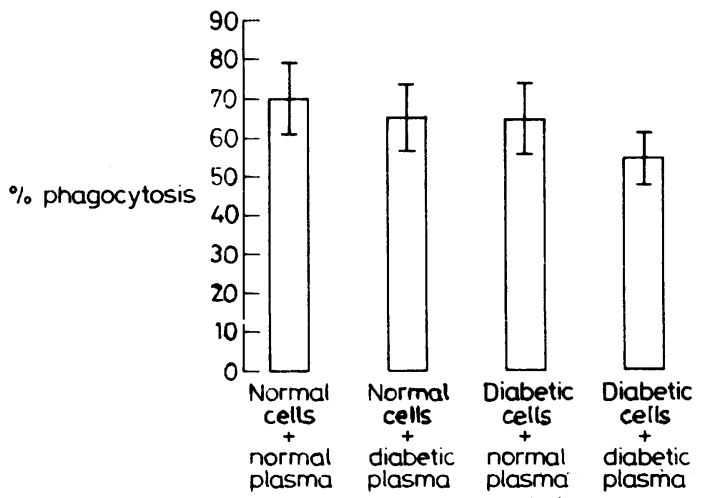

Fig. 2 Percentage phagocytosis after incubation for $5 \mathrm{~min}$ in mixtures of diabetic and normal cells and plasma. Results shown as mean \pm 1 standard deviation. 


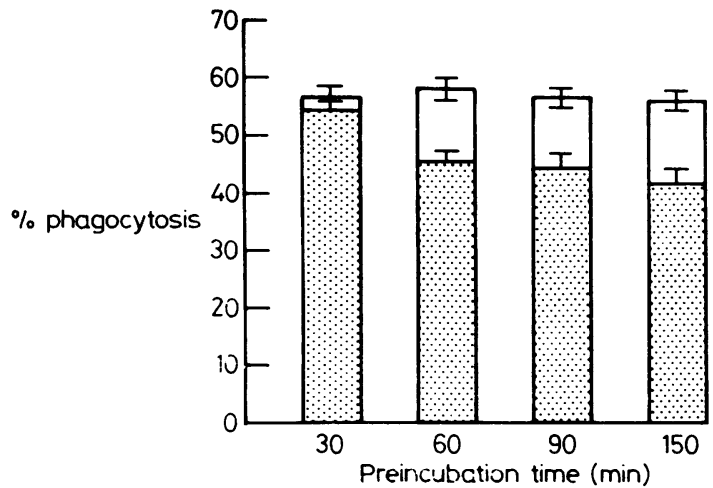

Fig. 3 Percentage phagocytosis after 5 min incubation in normal plasma which had been preincubated at $52 \mathrm{mmol} / \mathrm{l}$ $D$ glucose for the times shown (hatched areas). For the controls (clear areas) normal plasma was preincubated for the same times and adjusted to $52 \mathrm{mmol} / \mathrm{l} D$ glucose immediately before measuring phagocytosis. Results shown as mean \pm 1 standard deviation.

to diabetic cells appeared to add up to the size of the defect when diabetic cells in diabetic plasma were compared with normal cells in normal plasma (Fig. 2). When candida preopsonised with normal plasma and washed was added to diabetic plasma and normal neutrophils there was no significant difference in phagocytosis compared with preopsonised candida in normal plasma $(69.5 \% \pm 5.0 \mathrm{SD}$ in diabetic plasma, $70.8 \% \pm 2.4 \mathrm{SD}$ in normal plasma after 5 min incubation). This experiment was repeated three times with the same result and indicates that the defect in diabetic plasma is due to deficient opsonisation.

In an attempt to reproduce the diabetic abnormality in vitro, normal neutrophils and plasma were incubated at high concentrations of D-glucose before measuring phagocytosis. Unfortunately difficulties with maintaining the glucose concentration and preventing cell clumping obscured any impairment of phagocytosis by preincubated neutrophils. When plasma was adjusted to a D-glucose concentration of $52 \mathrm{mmol} / \mathrm{l}$ immediately before measuring phagocytosis there was no impairment. After 30 min preincubation of normal plasma at a D-glucose concentration of $52 \mathrm{mmol} / 1$ at $37^{\circ} \mathrm{C}$ there was still no significant impairment of phagocytosis, but by $60 \mathrm{~min}$ there was a significant defect which increased as the duration of preincubation increased (Fig. 3). The same concentration of the inert sugar D-mannitol had no effect. Preincubation of normal plasma at lower concentrations of D-glucose for $180 \mathrm{~min}$ at $37^{\circ} \mathrm{C}$ resulted in impaired phagocytosis, which was related to the concentration of D-glucose (Fig. 4). At a D-glucose concentration of $15 \mathrm{mmol} / \mathrm{l}$

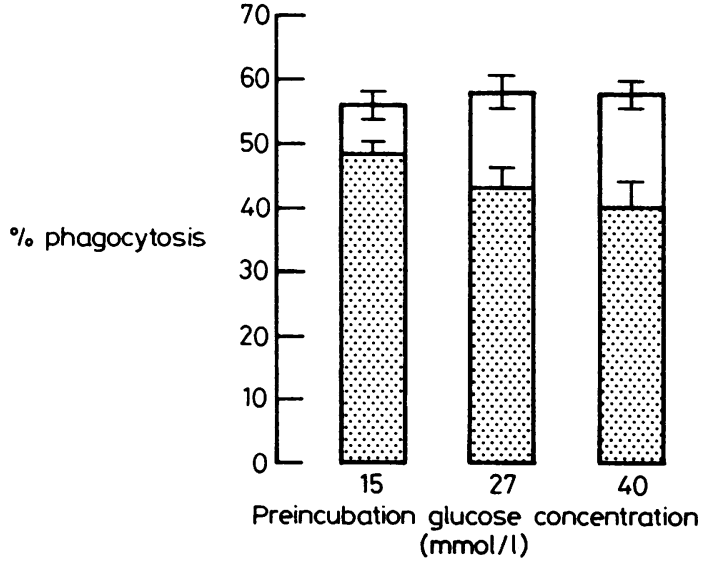

Fig. 4 Percentage phagocytosis after 5 min incubation in normal plasma which had been preincubated for $180 \mathrm{~min}$ in $D$ glucose at the concentrations shown (hatched areas). For the controls (clear areas) normal plasma was preincubated for 180 min and the $D$ glucose concentrations adjusted immediately before measuring phagocytosis. Results shown as mean \pm 1 standard deviation.

the size of the defect was similar to that seen with diabetic plasma.

\section{Discussion}

The tendency of patients with diabetes mellitus to develop a variety of infections may be due to defects in host defences. Studies in animals rendered diabetic and humans with diabetes have confirmed abnormal neutrophil adherence, chemotaxis, phagocytosis, and microbial killing. ${ }^{2-7}$ The mechanisms responsible for these defects are not certain, but in general they correlate with poor control of the diabetes and are corrected by reversal of the hyperglycaemia, hyperosmolarity, and ketoacidosis with insulin. ${ }^{6}$ There remains controversy as to whether neutrophil function and particularly phagocytosis is abnormal in uncomplicated diabetics. ${ }^{3-6}$ Control of diabetes is relative; ideally the blood sugar should be kept within the normal range all the time, but this is not yet possible. Different levels of diabetic control may account for some of the discrepant results reported, but differences in methods for measuring phagocytosis are probably more important. We studied a group of uncomplicated diabetic outpatients treated with insulin whose control, as indicated by random blood sugar measurements and the level of glycosylated haemoglobin, was well within the usual experience of a diabetic clinic. Our method for measuring phagocytosis entailed arranging the ratio of yeasts to neutrophils so that within $30 \mathrm{~min}$ 
well over $90 \%$ of normal neutrophils would have ingested one or more organisms (Fig. 1). In particular we looked at the beginning of the incubation period, when phagocytosis is proceeding rapidly, and found considerable and significant impairment in the diabetics.

Mixing experiments with diabetic cells in normal plasma and vice versa showed a defect in both the cells and the plasma. It therefore seems likely that the mechanism is a reaction between glucose and proteins concerned with phagocytosis; in plasma these are $\mathrm{C} 3 \mathrm{~b}$ and the Fc component of immunoglobulin and on the neutrophil the surface receptors for these opsonins. ${ }^{12}$ Unfortunately, for technical reasons we were unable to show a reaction between glucose and neutrophils, but we did show a reaction between plasma proteins and glucose leading to impaired phagocytosis. Furthermore, this impairment could be produced by incubation of plasma at glucose concentrations $(15 \mathrm{mmol} / \mathrm{l})$ which occur in diabetics treated with once or twice daily insulin injections. We failed to find a correlation between impaired phagocytosis and the level of glycosylated haemoglobin, but we looked at only a small number of patients and a close correlation should not be expected as the half life in the circulation of immunoglobulin, complement, and neutrophils are different and very different from that of haemoglobin.

It is impossible to quantify the relation between impaired phagocytosis and the tendency of diabetics to develop infections. Indeed, infections may not be very common in otherwise uncomplicated diabetics, ${ }^{13}$ but when infections do occur in diabetics they are a serious problem with a high mortality and good control of the blood sugar seems to be an essential part of treatment. ${ }^{14} \mathrm{~A}$ relation between phagocytosis and the blood sugar concentration might explain the observations. It is also interesting to speculate how impaired phagocytosis might contribute to other complications such as atherosclerosis of arteries and small vessel lesions in the retina and kidneys. It is becoming clear that removal of dead or damaged cells requires opsonisation by immunoglobulin before removal by mononuclear phagocytes, and this process has many similarities to neutrophil phagocytosis of invading micro-organisms. ${ }^{15}$ Thus a small impairment of phagocytic function due to reaction of proteins with higher than normal concentrations of plasma glucose could lead to the gradual accumulation of lipid and other cell debris, which may be deposited in vessel walls and so con- tribute to long term complications of diabetes. Such a mechanism might also be important in subjects who, while not overtly diabetic, have some impairment of glucose tolerance due, for example, to obesity, as reactions between glucose and proteins are not confined to diabetics.

We thank Dr W Jeffcoate for kindly allowing us to study his patients.

\section{References}

Silva J, Fekety FR Jr. Acute complications of the diabetic state. In: Fajans SS, ed. Diabetes mellitus. Washington DC: US Government Printing Office, 1976:154-70.

${ }^{2}$ Bagdade JD, Stewart M, Walters E. Impaired granulocyte adherence: $A$ reversible defect in patients with poorly controlled diabetes. Diabetes 1978;27:677-81.

${ }^{3}$ Miller ME, Baker L. Leukocyte function in juvenile diabetes mellitus: Humoral and Cellular Aspects. J Pediatr 1972;81:979-82.

${ }^{4}$ Molenaar DM, Palumbo PJ, Wilson WR, Ritts RE. Leukocyte chemotaxis in diabetic patients and their non diabetic first degree relatives. Diabetes 1976;25(suppl 2):880-3.

s Bagdade JD, Nielson KL, Bulger RJ. Reversible abnormalities in phagocytic function in poorly controlled diabetic patients. Am J Med Sci 1972;263:451-6.

- Bagdade JD, Root RK, Bulger RJ. Impaired leukocyte function in patients with poorly controlled diabetes. Diabetes 1974;23:9-15.

${ }^{7}$ Nolan CM, Beaty HN, Bagdade JD. Further characterisation of the impaired bactericidal function of granulocytes in patients with poorly controlled diabetes. Diabetes 1978;27:889-94.

Bybee JD, Roger DE. The phagocytic activity of polymorphonuclear leukocytes obtained from patients with diabetes mellitus. J Lab Clin Med 1964;64:1-13.

' Crosby B, Allison F. Phagocytic and bactericidal capacity of polymorphonuclear leukocytes recovered from venous blood of human beings. Proc Soc Exp Biol Med 1966; 123:660-4.

10 Tan JS, Anderson JL, Watankunakom C, Phair JP. Neutrophil dysfunction in diabetes mellitus. $J$ Lab Clin Med 1975;85:26-33.

" El Maallem H, Fletcher J. Defective neutrophil function in chronic granulocytic leukaemia. Br $J$ Haematol 1976;34:95-103.

12 Scribner DJ, Fahrney D. Neutrophil receptors for IgG and complement: Their roles in the attachment and ingestion phases of phagocytosis. $J$ Immunol 1976;116:892-7.

${ }^{13}$ Munro JF. Diabetes and infection. J Antimicrob Chemother 1976;2:322-4.

14 Wheat LJ. Infection and diabetes mellitus. Diabetic Care $\mathrm{N}$ 1980;3: 187-97.

is Kay MMB. Mechanism of removal of senescent cells by human $N$ macrophages in situ. Proc Natl Acad Sci USA 1975; 72:3521-5.

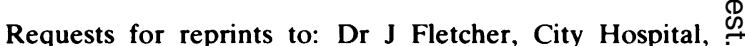
Hucknall Road, Nottingham NG5 1PB, England. 